\title{
Shape-Preserving Accelerating Electromagnetic Wave Packets in Curved Space
}

\author{
Rivka Bekenstein, Jonathan Nemirovsky, Ido Kaminer, and Mordechai Segev \\ Physics Department and Solid State Institute, Technion, Haifa 32000, Israel \\ (Received 7 August 2013; revised manuscript received 6 February 2014; published 13 March 2014)

\begin{abstract}
We present shape-preserving spatially accelerating electromagnetic wave packets in curved space: wave packets propagating along nongeodesic trajectories while periodically recovering their structure. These wave packets are solutions to the paraxial and nonparaxial wave equations in curved space. We analyze the dynamics of such beams propagating on surfaces of revolution, and find solutions that propagate along a variety of nongeodesic trajectories, with their intensity profile becoming narrower (or broader) in a scaled self-similar fashion. Such wave packets reflect the interplay between the curvature of space and interference effects. Finally, we extend this concept to nonlinear accelerating beams in curved space supported by the Kerr nonlinearity. Our study concentrates on optical settings, but the underlying concepts directly relate to general relativity.
\end{abstract}

DOI: 10.1103/PhysRevX.4.011038

The complex dynamics of particles and of electromagnetic (EM) waves in curved space-time is still inaccessible to laboratory experiments. However, numerous physical systems have been suggested to demonstrate analogies of general-relativity phenomena, ranging from sound and gravity waves in flowing fluids [1-3] to Bose-Einstein [4-6] and optical systems, which have had a major success in demonstrating such phenomena [7-13]. For example, metamaterials enabled creating analogies to black holes, by engineering the $(\mathrm{EM})$ properties of the material through which light is propagating [8-10]. Another example is using a moving dielectric medium that acts as an effective gravitational field on the light [12]. This idea was demonstrated experimentally by employing ultrashort pulses in an optical fiber to create an artificial event horizon [13]. Another route for such studies is to create curved space by engineering the geometry of the space itself. This idea, suggested in 1981 [14], started by exploring the dynamics of a free quantum particle constrained by an external potential to evolve within a thin sheet. More than 25 years later, these ideas were carried over to EM waves [15], where pioneering experiments studied light propagating in a thin-film waveguide attached to the curved surface area of a three-dimensional (3D) body [16]. However, thus far, in all of these experiments and theoretical studies on general-relativity concepts with EM waves, the wave packets were propagating on geodesic trajectories, which are naturally the shortest path, analogous to straight lines in flat geometry. But, do wave packets propagating in curved space have to follow special geodesic paths, or can they

Published by the American Physical Society under the terms of the Creative Commons Attribution 3.0 License. Further distribution of this work must maintain attribution to the author(s) and the published article's title, journal citation, and DOI.
Subject Areas: Optics

exhibit other trajectories that are not predicted by the geodesic equation?

Here, we show that wave packets can exhibit periodically shape-invariant spatially accelerating dynamics in curved space, propagating in nongeodesic trajectories that reflect the interplay between the curvature of space and interference effects arising from initial conditions. We study these beams in surfaces of revolution in the linear and nonlinear, paraxial and nonparaxial regimes and unravel a variety of new intriguing properties that are nonexistent in flat space. This study paves the way to accelerating-beams experiments in curved space to study basic concepts of general relativity, where the entire dynamics is nongeodesic.

Before proceeding, we briefly recall the ideas underlying accelerating wave packets. They were first revealed in 1979 as a unique solution to the Schrödinger equation: a propagation-invariant wave packet shaped as an Airy function that accelerates in time [17]. Almost 30 years later, the concept of accelerating wave packets was introduced into electromagnetism, demonstrating Airy beams that are spatially accelerating within the paraxial approximation $[18,19]$. Following Refs. $[18,19]$, accelerating wave packets have drawn extensive interest and initiated many new ideas, such as accelerating ultrashort pulses and light bullets [20-22], two-dimensional (2D) accelerating beams [23], accelerating beams following arbitrary convex acceleration trajectories [24-26], accelerating beams in photonics potentials [27-30], and accelerating beams in nonlinear media [31-34]. These recent works were followed by many applications such as manipulating microparticles [35,36], self-bending plasma channels [37], and accelerating electron beams [38]. For some time, shape-preserving accelerating wave packets were believed to exist strictly within the domain of the Schrödinger-type paraxial wave equation [17-19]. However, in 2012, we presented accelerating 
shape-invariant wave packets that are exact solutions of Maxwell's equations [39]. Experimental demonstrations of such beams followed soon thereafter [40-42], along with further theory and experiments demonstrating additional families of nonparaxial accelerating beams [43-45]. Thus far, however, accelerating wave packets remained strictly within the realm of flat space.

Since the dynamics of EM waves in curved space is significantly different from that in flat space, a natural question to ask is whether accelerating wave packets can exist at all in curved space, and if they do, how do their features differ from those in flat space? In other words, are there wave packets that travel along nongeodesic trajectories in free space without contradicting the basic concepts of general relativity?

Consider EM waves that are restricted to exist in a 2D curved surface. This physical situation can be achieved by covering the surface area of a 3D shape (a sphere, for example) with a thin homogenous layer of a material with a higher refractive index. Such a layer acts as a waveguide, keeping the light confined inside it due to total internal reflection (Fig. 1). The dynamics of EM fields in curved space can be described by the 3D Maxwell equations in general coordinates [46]:

$$
\begin{aligned}
\frac{1}{2 \sqrt{g}} \varepsilon^{\alpha \beta \gamma}\left(\frac{\partial E_{\gamma}}{\partial x^{\beta}}-\frac{\partial E_{\beta}}{\partial x^{\gamma}}\right)+\frac{1}{c} \frac{\partial B^{a}}{\partial t} & =0, \\
\frac{1}{\sqrt{g}} \frac{\partial}{\partial x^{a}}\left(\sqrt{g} B^{a}\right) & =0, \\
\frac{1}{2 \sqrt{g}} \varepsilon^{\alpha \beta \gamma}\left(\frac{\partial H_{\gamma}}{\partial x^{\beta}}-\frac{\partial H_{\beta}}{\partial x^{\gamma}}\right)-\frac{1}{c} \frac{\partial D^{a}}{\partial t} & =0, \\
\frac{1}{\sqrt{g}} \frac{\partial}{\partial x^{a}}\left(\sqrt{g} D^{a}\right) & =0 .
\end{aligned}
$$

Here, $g$ is the time-independent spatial metric determinant, where $d s^{2}=g_{\alpha \beta} d x^{\alpha} d x^{\beta}$ (the spatial indices $\alpha, \beta$, and $\gamma$ run from 1 to 3$), \varepsilon^{\alpha \beta \gamma}$ is the antisymmetric Levi-Civita tensor, and $E^{\alpha}, H^{\alpha}, D^{\alpha}$, and $B^{\alpha}$ are three-vectors. The wave equation for the electric field is derived from Eqs. (1) [15]:

$$
\begin{aligned}
& -\frac{1}{\sqrt{g}} \partial_{\beta} \sqrt{g} g^{\beta \gamma} \partial_{\gamma} E^{\alpha}+\frac{1}{\sqrt{g}} \partial_{\beta} \sqrt{g} g^{\alpha \gamma} \partial_{\gamma} E^{\beta}+\frac{1}{c^{2}} \frac{\partial^{2} E^{\alpha}}{\partial t^{2}} \\
& =-\frac{1}{c^{2}} \frac{\partial^{2} P^{\alpha}}{\partial t^{2}}
\end{aligned}
$$

where the polarization $P^{\alpha} \equiv D^{\alpha}-E^{\alpha}$ can generally be nonlinear in the electric field. Notice that the second term does not appear in homogenous flat space: It arises strictly due to the curved-space geometry.

We are interested in the evolution of the electric field in a general surface of revolution. First, we introduce the metric of such a surface. These surfaces are parametrized by $\vec{s}(u, v)=[\alpha(u) \cos (v), \alpha(u) \sin (v), \beta(u)]$, where $v=$ $[-\pi, \pi]$ is the angle of rotation and $-\infty<u<\infty$ is a general parametrization of the surface along its axis of the revolution. Every point in $3 \mathrm{D}$ space $(\vec{r})$ can be described by the two coordinates on the curved surface $(u, v)$ and a third coordinate $(h)$ normal to the surface at every point: $\vec{r}(u, v, h)=\vec{s}(u, v)+h \vec{N}(u, v)$, where $\vec{N}(u, v)$ is the unit vector normal to the surface (Fig. 1). We transform to a new set of coordinates: $z=\int_{0}^{z} \sqrt{\alpha^{\prime 2}(u)+\beta^{\prime 2}(u)} d u$ and $x=R_{0} v$, where $R_{0}$ is defined by the radius of the surface at $z=0, R_{0}=\alpha(0)$, and $x$ has units of length in the transverse direction at $z=0$. The metric takes the form $d l^{2}=d z^{2}+\left\{\alpha^{2}[u(z)] / R_{0}^{2}\right\} d x^{2} \triangleq d z^{2}+\gamma d x^{2}$, where $\gamma$ is defined as the dimensionless 2D metric determinant. As in Ref. [15], we decouple the wave equation for the different polarizations [14]. This procedure can be done for surfaces that have small enough Gaussian and mean curvatures, and that their mean curvature varies on scales that are large compared with the wavelength. For example, for a wavelength in the visible range, the radius of curvature of such a
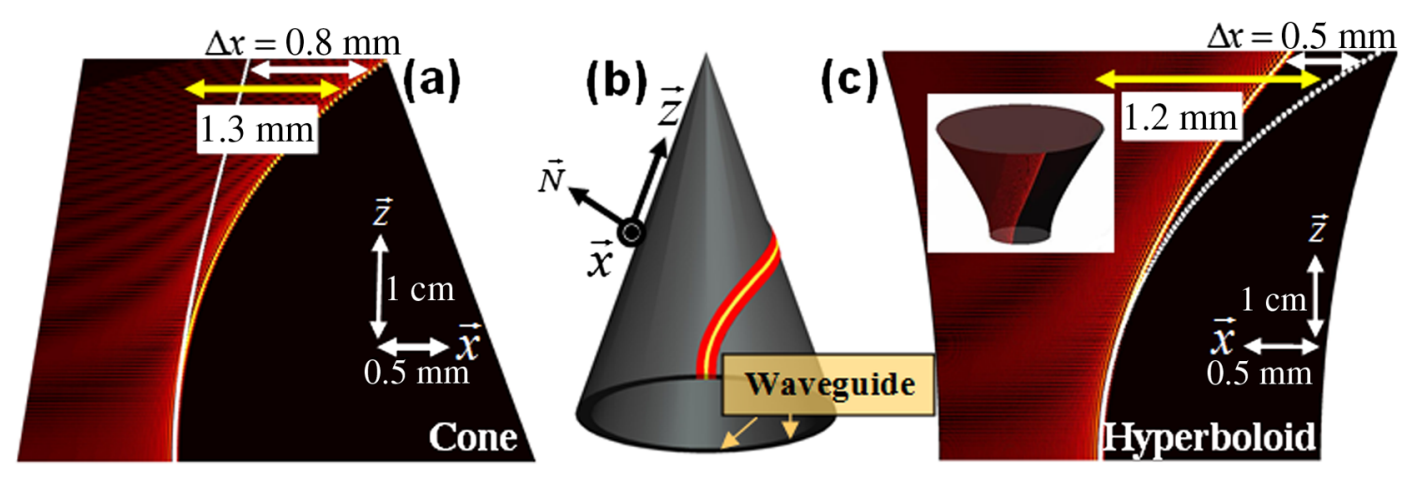

FIG. 1. (b) Sketch of a surface of revolution (cone). The EM field is restricted to propagate within the surface area by a thin waveguide layer. (a)-(c) The evolution of the envelope of an accelerating beam ( $\psi$ ) on the surface area of a cone (a) and of an hyperboloid (both with $10-\mathrm{cm}$ height, $3-\mathrm{mm}$ base radius, and a propagation constant of $1.2 e 7 \mathrm{~m}^{-1}$ ). (c) The dashed white line displays the propagation of the same beam in flat space, projected on the surface of revolution. The beam apertures are (a) $9 \mathrm{~mm}$ and (c) $6 \mathrm{~mm}$, with main lobes of widths of (a) $30 \mu \mathrm{m}$ and (c) $33 \mu \mathrm{m}$. 
surface has to be of the order of millimeters, a regime in which practically all macroscopic optical components exist. (The exceptions are microlenses, microcavities, single-mode fibers, etc.) We are interested in waves propagating in the $z$ direction. The simplest cases are the transverse electric (TE) modes, for which the electric field has no $z$ component; hence, they are $x$ polarized, in the form $\vec{E}(z, x, h)=[0, \phi(z, x) \xi(h), 0]$, which yields

$$
\begin{gathered}
\frac{1}{\gamma} \partial_{x}^{2} \phi+\partial_{z}^{2} \phi+\frac{\sqrt{\gamma}}{\sqrt{\gamma}} \partial_{z} \phi+q^{2} \phi+V_{\mathrm{NL}}(\phi) \phi=0, \\
-\partial_{h}{ }^{2} \xi-k_{0}{ }^{2} n_{0}{ }^{2} \xi=-q^{2} \xi
\end{gathered}
$$

Here, $n_{0}$ is the refractive index in the surface layer, $k_{0}$ is the vacuum wave number, and $q$ has units of $1 / \mathrm{m}$.

The boundary conditions here yield some unexpected implications. Naturally, beams propagating on surfaces of revolution must fulfill periodic boundary conditions for every $z$. Thus, we first find solutions in an infinite space and then use their superpositions to construct solutions satisfying periodic boundary conditions. This methodology serves as a powerful tool to find the solutions in the linear regime $\left[V_{\mathrm{NL}}(\phi)=0\right]$ where superposition holds. To do that, we use the universal covering space: a covering map of an infinite 1D space mapped to a ring on the surface (points having the same $z$. Each point on the surface is an image of an infinite number of points located in the universal covering space. We use the covering map to construct solutions as follows:

$$
\phi_{p}\left(z, x_{p}\right)=\sum_{m=-\infty}^{\infty} \phi\left(z, x+2 \pi m R_{0}\right) .
$$

$\phi_{p}$ is a solution of Eq. (3a) satisfying periodic boundary conditions, where $x_{p}, x \in[0,2 \pi]$. Equation (4) reflects the fact that Eq. (3a) is linear in $\phi$ [that is, when $V_{\mathrm{NL}}(\phi)=0$ ].

First, we focus on the paraxial regime and derive the equation for the slowly varying amplitude $\psi(z, x)$, assuming that $\left|\partial^{2} \psi / \partial z^{2}\right| \ll|2 q \partial \psi / \partial z|$. We use the ansatz $\phi(z, x)=\frac{1}{\sqrt{\gamma}} \psi(z, x) e^{i q z}$, where the field amplitude $\phi(z, x)$ varies with the algebraic factor $\sqrt{\gamma}$, for the power to be conserved. This ansatz yields the paraxial equation for a general surface of revolution:

$$
2 i q \partial_{z} \psi=-\frac{1}{\gamma} \partial_{x}{ }^{2} \psi-V_{\mathrm{eff}}(z) \psi-V_{\mathrm{NL}}(\psi) \psi,
$$

where the effective one-dimensional potential depends on the determinant of the surface $V_{\text {eff }}(z)=\left[\sqrt{\gamma} /(1 / \sqrt{\gamma})_{z}\right]_{z}$. Clearly, the paraxial equation describing the propagation of EM waves within surfaces of revolution involves more complex evolution than the propagation of an optical beam in flat space. First, the surface curvature acts as a $z$-dependent one-dimensional potential even for homogeneous surfaces. Second, the spatial frequencies vary during propagation, in analogy to the redshift and blueshift occurring in curved space-time. Consequently, the shapes of the eigenmodes describing the waves propagating in such a surface evolve when the curvature of space varies during propagation.

We seek an accelerating solution to Eq. (5), namely, a solution that is propagation invariant in the accelerating frame of reference. Such solutions should satisfy $|\psi(0, x)|=|\psi[z, x-f(z)]|$, meaning that the beam would propagate along the curve $x=f(z)$ while maintaining its intensity structure. We want to transform Eq. (5) to the paraxial equation in flat space and use the known solution of the accelerating Airy beam. To do that, we first cancel the effective potential using a gauge transformation $\tilde{\psi}=\psi e^{-(i / 2 q)} \int_{0}^{z} V_{\text {eff }}\left(z^{\prime}\right) d z^{\prime}$. Then, we use a transformation of coordinates of the form $\tilde{z}=\int_{0}^{\tilde{z}} \frac{1}{\gamma\left(z^{\prime}\right)} d z^{\prime}$ and find the accelerating beam in curved space to be

$$
\begin{aligned}
\psi(z, x)= & \operatorname{Airy}[x-f(z)] \\
& \times \exp \left[i\left(\frac{a}{2 q} \int_{0}^{z} \frac{1}{\gamma} d z^{\prime}\right)[x-f(z)]\right. \\
& \left.+\frac{i}{2 q} \int_{0}^{z} V_{\text {eff }}\left(z^{\prime}\right) d z^{\prime}\right]
\end{aligned}
$$

where $a$ is a constant with units $[a]=1 / \mathrm{m}^{3}$. The expression for the trajectory $[f(z)]$ of the Airy beam in curved space is given by

$$
f(z)=a\left(\frac{1}{2 q} \int_{0}^{z} \frac{1}{\gamma} d z^{\prime}\right)^{2} .
$$

Equation (7) defines acceleration trajectories that depend on the metric determinant. Consequently, the acceleration trajectory is different for every surface of revolution and can even become nonconvex in $x$, as shown in Fig. 1. Notice that, generally, the accelerating solution of Eq. (5) is not shape preserving because $|\psi|^{2}$ varies with $z$. However, it is self-similar and can become narrower or broader during propagation, according to the geometry of the specific surface [47].

To understand the origin of the nongeodesic trajectory, we introduce a particle model to describe the trajectory of the main lobe of the accelerating beam. We account for the interference effect through an inhomogeneous term in the geodesic equation:

$$
\frac{d^{2} x}{d \lambda^{2}}+\frac{\gamma_{z}}{\gamma} \frac{d x}{d \lambda} \frac{d z}{d \lambda}=\frac{\tilde{F}}{\sqrt{\gamma}},
$$

where $\lambda$ is an affine parameter that, in this case, can be the line element. This equation describes the motion of a particle in a surface of revolution under the influence of a 
force, where $\tilde{F}$ has the dimensions of force per unit of mass when $\lambda$ is taken to be time. Obviously, $\tilde{F}$ is a "fictitious" force because no real force is acting here. Constraining the motion of the particle to the paraxial regime $|d x / d z| \ll 1$ yields the approximate line element $d \lambda=d z\left\{1+\frac{1}{2} d x^{2} /\right.$ $\left.d z^{2}+O\left[\left(d x^{2} / d z^{2}\right)^{2}\right]\right\}$. To first order, Eq. (8) becomes

$$
\frac{d^{2} x}{d z^{2}}+\frac{\gamma_{z}}{\gamma} \frac{d x}{d z}=\frac{\tilde{F}}{\sqrt{\gamma}} .
$$

Here, the "fictitious" force $\tilde{F}=a / k(\sqrt{\gamma})^{3}$, which manifests the interference effect induced by the structure of the wave packet and also reflects the dependence on the curvature of the surface of revolution. This "fictitious" force is a unique wave phenomenon that a particle model cannot describe. While propagating on a surface of revolution, the arc length is stretched as $d \lambda=\sqrt{\gamma} d x$; hence, the spatial frequencies of the beam are stretched with an opposite trend. This stretching changes the "fictitious" force $\tilde{F}$ by a factor of $(\sqrt{\gamma})^{3}$, as can be seen directly from the cubic phase of the accelerating beam in $k$ space. The solution for $x(z)$ in Eq. (9) is exactly the trajectory of the Airy beam $f(z)$, from Eq. (7).

The nonparabolic acceleration trajectories in curved space can be understood by examining Eq. (9), which manifests the interplay between the effect of the curvature and the effect of interference. The right-hand side comes from the interference effect, acting as if an effective potential exerts a "fictitious" force on the wave packet. In flat space, the second term on the left is zero because $\gamma=$ const, and the equation becomes the Newton equation for a particle under a constant force, which yields a parabolic trajectory. The same parabolic trajectory is the trajectory of the Airy beam in flat space. Clearly, the curvature of space has a major effect on the trajectory of the beam, through the "fictitious" force. However, the curvature

also gives rise to another term in Eq. (9): the second term $\gamma_{z} / \gamma$, which is one of the two nonzero Christoffel symbols.

Thus far, we have generalized the paraxial accelerating beam to curved space and showed the various trajectories possible that are not the natural geodesics of these surfaces, but we have not found the actual solutions as of yet. To do that, we construct a beam that propagates on the trajectories defined by Eq. (7) and also fulfills periodic boundary conditions, as necessary for surfaces of revolution. Such solutions are naturally periodic [48] and they are obtained from the Airy solution defined on the universal covering space, using Eq. (4):

$$
\begin{aligned}
\psi\left(z, x_{p}\right)= & \sum_{m=-\infty}^{m} C_{m} \exp \left(\frac{i k_{m}^{3}}{3 a}+i k_{m} x\right) \\
& \times \exp \left\{i\left[\left(\frac{a}{2 q} \int_{0}^{z} \frac{1}{\gamma} d z^{\prime}\right) x+\frac{1}{2 q} \int_{0}^{z} V_{\mathrm{eff}}\left(z^{\prime}\right) d z^{\prime}\right]\right\},
\end{aligned}
$$

where $k_{m}=m / R_{0}$ and $m$ is an integer. The initial beam (the beam at $z=0$ ) is actually an infinite Airy beam that is wrapped on a circular perimeter, over and over again. This solution satisfies Eq. (5) and also the periodic boundary conditions. These conditions can be satisfied only by specific (quantized) values of transverse momentum. Hence, the beam is composed of a discrete set of "spectral functions." To stay within paraxiality, we limit the spatial spectrum from above, by setting $C_{m}=0$ for every spatial frequency above the $k_{M}$ defining the boundary of the paraxial regime. Importantly, the number of these spectral functions comprising the beam is constrained both from below and from above: The lowest transverse wave number that can be excited when the beam is launched (at $z=0$ ) is $k_{1}=1 / R_{0}$, while the highest $k_{M}=M / R_{0}$ occurs for $m=M$. This finite range within which the spatial frequencies of the accelerating beam can exist has immediate physical consequences: Such a curved-space accelerating beam carries finite power because it is constrained to a circular perimeter and constructed from a finite number of spatial frequencies, due to the cutoff. This finding has an important implication: Having a finite power, one can now define a center of mass for the accelerating beam. It is important to emphasize that although the self-reconstructing structure of the wave packet travels along a nongeodesic trajectory, the center of mass travels along a geodesic trajectory as in Refs. $[17,18]$. However, almost all the applications of accelerating beams rely on light-matter interactions, where the important parameter is the local intensity and not the center of mass, e.g., acceleration of particles [35], formation of curved plasma channels [37], laser machining [49], to name a few out of many. For all such applications, what matters is the accelerating main lobe where the intensity is the highest, while the fact that the center of mass is propagating on a straight line is unimportant.

In examining the structure of the curved-space accelerating beam, we notice that it can be different from the Airy beam, whose envelope is monotonically decaying. Here, the shape-preserving wave packet accelerating in curved space can have several parallel beams whose number is set by the initial choice of the spectral components $C_{m}$.

The accelerating solution in curved space is propagating on the curve defined by Eq. (7) and is periodically shape invariant: It recreates its exact intensity profile in $x_{p}$ for discrete $z$ values defined by $\int_{0}^{z_{q}} \frac{1}{\gamma} d z^{\prime}=\frac{2 q l}{a R_{0}}$, where $l$ is an integer. Notice the nonconstant spacing between planes of self-reconstruction that depends on the curvature of space. This interesting feature results from the transverse momentum being quantized (rather than continuous). Interestingly, $a$ also determines the curvature of the trajectory: The faster the beam accelerates, the faster it recreates itself.

After having presented the paraxial accelerating beams in curved space and their properties, we now proceed to the nonparaxial beams that are solutions of Maxwell's 
equations on surfaces of revolution. We begin with Eq. (3a), which describes the linear nonparaxial regime. We apply a transformation of coordinates that simplifies the equation for any surface of revolution. We set $Z=\int_{0}^{z} \frac{1}{\sqrt{\gamma\left(z^{\prime}\right)}} d z^{\prime}$, which yields

$$
\partial_{x}^{2} \phi+\partial_{Z}^{2} \phi+\gamma q^{2} \phi=0
$$

Clearly, the nonparaxial case is more complicated than the paraxial one: Eq. (11) is essentially the Helmholtz equation with a $z$-dependent refractive index. Equation (15) allows back propagation and back reflections. Here, we look only for a forward-propagating wave packet. Since we cannot solve at this point for the most general case, we examine three generic solutions that allow for close-form solutions. The first case of a surface of revolution is a cylinder, where the metric determinant is not $z$ dependent: $\gamma(z)=1$. The solution in the covering space coincides with the form of the solution in flat space, which is described in detail in Ref. [39]. Using the same method we used for the paraxial beam [Eq. (4)], we construct the accelerating wave packet:

$$
\hat{\phi}\left(Z, x_{p}\right)=\sum_{q_{n}} D_{n} \exp \left\{i \beta q_{n}+i q\left[x_{p} \cos \left(q_{n}\right)+Z \sin \left(q_{n}\right)\right]\right\}
$$

We choose $D_{n}=0$ for any $q_{n}$ that is not between 0 and $\pi$, meaning that we allow only forward-propagating waves (i.e., we assume that the backward-propagating waves are not excited at $z=0$ ). This wave packet is constructed from a discrete set of spatial frequencies that fulfill the periodic boundary conditions: $q_{n}=\arccos \left(n / q R_{0}\right)$ (see Fig. 2). The spectrum is now limited from above because at a high enough spatial frequency, the propagation constant becomes imaginary and the spectral function becomes evanescent. Here, we are not interested in the evanescent waves; hence, we set their initial population to zero $\left(D_{n}=0\right.$ for those modes). This nonparaxial accelerating beam carries finite power. In fact, the solution can support several parallel beams accelerating (bending) in parallel, as in the paraxial case, for a suitable choice of $D_{n}$. As for the nonparaxial flat-space accelerating beams [39], this nonparaxial curved-space wave packet is approximately shape invariant because it is a superposition of only forwardpropagating waves $\left(0<q_{n}<\pi\right)$. If the counterpropagating waves were to be taken in the superposition, the beam would have been fully shape preserving. Nevertheless, this wave packet (Fig. 2) accelerates on a circular trajectory while bending to very large (almost $90^{\circ}$ ) nonparaxial angles. The beam reconstructs itself in discrete angles in the $x, z$ plane, specifically for $\theta_{n}=\arccos \left(n / q R_{0}\right)$. We point out, however, that as $R_{0}$ becomes smaller, there are fewer propagating modes, and the acceleration that is an outcome of the interference between the modes decreases. Eventually, when $R_{0}$ becomes smaller than the wavelength of the light, all the excited spatial functions become evanescent. Equation (12) defines a family of solutions for a given trajectory, where every $\beta$ gives a beam with a different structure. Thus, every superposition of such beams (of various values of $\beta$ ) also forms a periodically shape-preserving accelerating beam.

Having solved for the simplest nonparaxial surface of revolution (a cylinder, where the metric is not $\mathrm{z}$ dependent), the natural question to ask is whether a nonparaxial

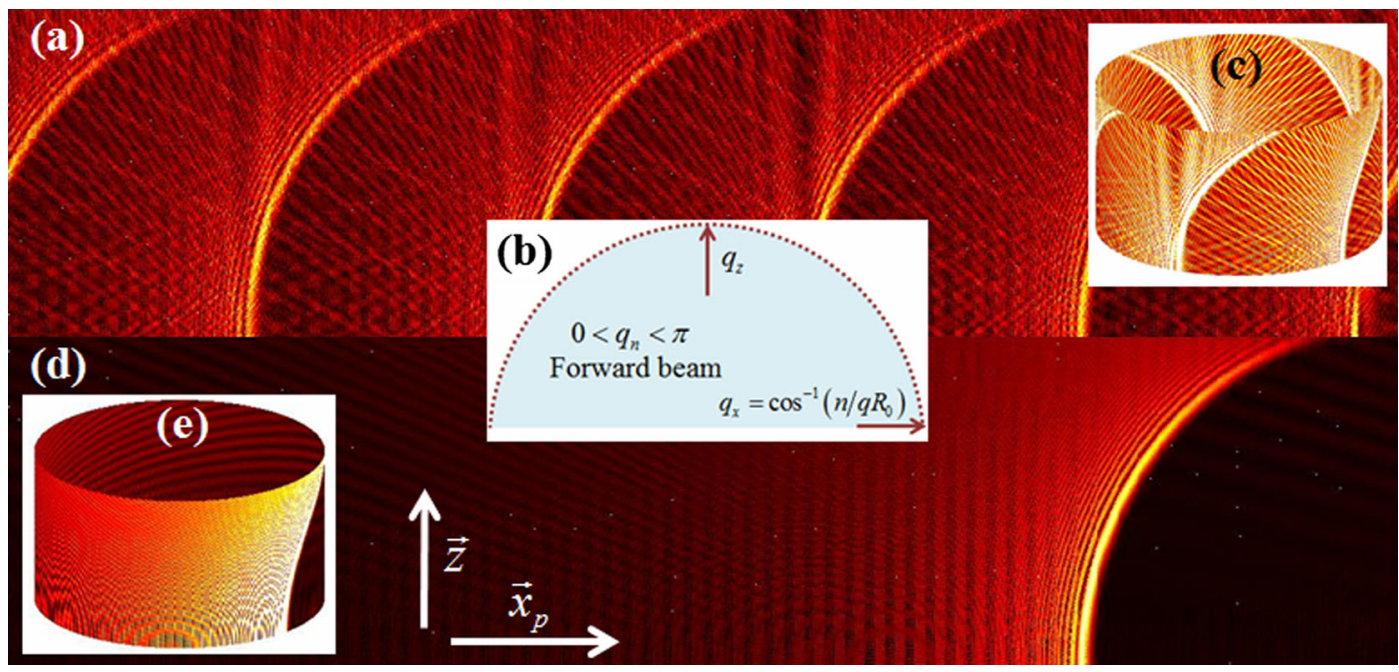

FIG. 2. Periodic accelerating beams constructed from discrete spatial frequencies as they propagate on various cylinders. This wave packet travels along circular trajectories while bending to very large nonparaxial angles. (a) The wave packet consists of several parallel beams that depend on the initial choice of $D_{n}$; only every fifth spectral function is populated, each with $D_{5 n}=1$. (b) Schematic illustration of the periodic accelerating beam in $k$ space. The beam is constructed from discrete spatial frequencies that reside in a halfcircle in $k$ space. This beam is a superposition of only forward-propagating waves. (d) Periodic accelerating beam on a cylinder, displaying a single intense main lobe $\left(D_{n}=1\right)$. (c),(e) The beams from (a) and (d) propagating on a surface of a cylinder. 
accelerating shape-invariant solution can exist for surfaces with a z-dependent curvature. Finding these kinds of solution is especially challenging because they cannot rely on the symmetry between all space coordinates, since this symmetry is inherently broken. Going back to Eq. (3a), we simplify the equation using $\phi(z, x)=1 / \gamma^{1 / 4} \zeta(z, x)$, which cancels the term with the first derivative in respect to $z$, yielding

$\partial_{x}^{2} \zeta+\gamma \partial_{z}^{2} \zeta+\gamma\left\{\frac{1}{4}\left[(\sqrt{\gamma})_{z}\right]^{2} / \gamma-\frac{1}{2}(\sqrt{\gamma})_{z z} / \sqrt{\gamma}+q^{2}\right\} \zeta=0$.

This equation is a Helmholtz-type equation with two differences: (1) There is an additional term that gives a $z$-dependent addition to the effective wave number, and (2) the $z$-dependent metric multiplies all the terms except for the derivative with respect to $x$. We want to transform Eq. (13) to a Helmholtz equation with a constant effective wave number. For this cause, we choose two specific surfaces, one with positive curvature and one with negative curvature, that will give a constant effective wave number: $\gamma_{p}=\cos ^{4}(\kappa z), \gamma_{n}=\cosh ^{4}(\kappa z)$. Equation (13) then simplifies to

$$
\partial_{x}^{2} \zeta / \gamma+\partial_{z}^{2} \zeta+\left(q^{2} \pm \kappa^{2}\right) \zeta=0
$$

where the \pm sign stands for the positive and negative curvatures, respectively. Following the same approximation regarding the slow change in curvature on the scale of a wavelength, we assume that $\kappa \ll 1 / R_{0}$. We find the accelerating wave packets on these surfaces to be

$$
\begin{aligned}
\hat{\phi}\left(z, x_{p}\right)= & 1 /\left(\gamma_{1,2}\right)^{1 / 4} \sum_{q_{n}} D_{n} \exp \left\{i \beta q_{n}+i \sqrt{q^{2} \pm \kappa^{2}}\right. \\
& \left.\times\left[x_{p} \sqrt{\gamma_{1,2}} \cos \left(q_{n}\right)+z \sin \left(q_{n}\right)\right]\right\} .
\end{aligned}
$$

These accelerating solutions are traveling along a noncircular trajectory, bending to very large angles (as displayed in Fig. 3). These noncircular trajectories can be easily understood in $k$-space, where the transverse spatial frequencies vary while the beam is propagating in the $z$ direction. The change in the spatial frequencies can cause a propagating mode to become evanescent while propagating in $z$. When this disappearance of modes occurs, the wave packet is no longer shape invariant. This effect of propagating modes that become evanescent is similar to known effects in different optical settings [50,51]. However, the modes in our system display strong anisotropy in their structure (radial vs azimuthal); hence, our system can support modes with complex structured azimuthal information that become evanescent. One of the most fascinating features is that the trajectory can even flip to the other direction-and accelerate toward the direction of the other

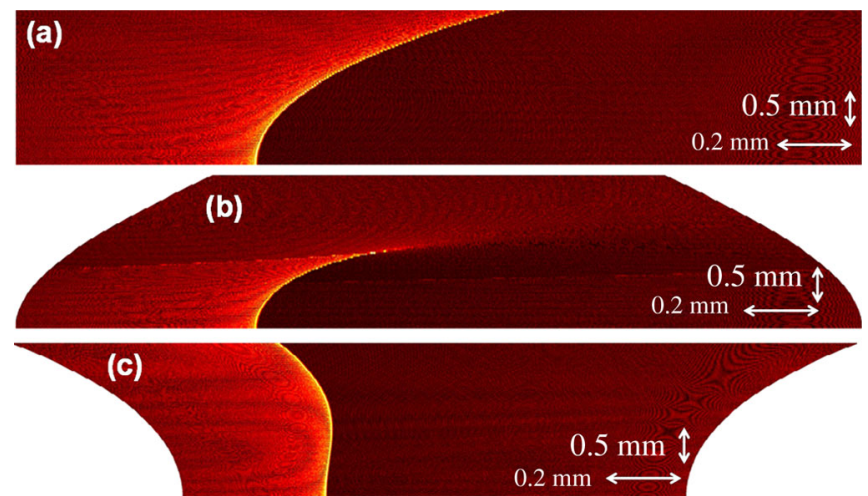

FIG. 3. Nonparaxial accelerating wave packets as they propagate on two kinds of surface, with (b) negative and (c) positive curvature. These wave packets propagate on various trajectories determined by the initial beam launched and the curvature of space. The propagation of the same beam on the surface of a cylinder is displayed in (a) for comparison. The spectral functions vary during propagation; in (b), at some propagation distance, the beam stops accelerating because all spectral frequencies become evanescent, and in (c), the trajectory of the beam becomes nonconvex. (The wavelength of the light in all figures is $0.5 \mu \mathrm{m}$.)

lobes (see Fig. 3). The reason is that the metric changes in the $z$ direction; however after some $\mathrm{z}$-value, the $\mathrm{z}$-direction is no longer the direction normal to the wave front, due to the nonparaxial trajectory. This interesting feature could not be seen in the paraxial case [52]. Naturally, this wave packet is also constructed only from a discrete set of spatial frequencies: $q_{n}=\arccos \left[n / \sqrt{\gamma\left(q^{2} \pm \kappa^{2}\right)} R_{0}\right]$. The discretization of the frequencies has a major impact on the profile of the wave packets, and it differs from that in flat space.

Finally, we return to the nonlinear case. For reasons of simplicity, we will deal here only with the paraxial regime. We seek a propagation-invariant solution of the paraxial nonlinear equation [Eq. (5)] traveling along a nongeodesic trajectory. Specifically, consider the Kerr effect, in which $V_{\mathrm{NL}}=\kappa|\psi|^{2} / \gamma$, where $\kappa$ is the effective nonlinear coefficient. We seek solutions (in the universal covering space) satisfying $|\psi(0, x)|=|\psi[z, x-f(z)]|$ and obtain an equation for the amplitude of the beam $u(\tilde{x})$ :

$$
\tilde{u}_{\tilde{x} \tilde{x}}-\tilde{x} \tilde{u}+\operatorname{sgn}(\kappa) \tilde{u}^{3}=0,
$$

where $\tilde{x}=\sqrt[3]{c}[x-f(z)]$ and $\tilde{u}=\kappa / c^{2 / 3} u$. The trajectory is the same as in the linear case [Eq. (7)]. We solve Eq. (16) numerically for the focusing and defocusing cases $(\kappa>0$ and $\kappa<0$, respectively). The only free parameters in our solution are the initial conditions. To find the wave function, we assume that the nonlinear accelerating beam decays for $\tilde{x} \rightarrow \infty$; thus, the nonlinear term in Eq. (16) is negligible for $\tilde{x} \rightarrow \infty$. Therefore, we choose the initial condition to be $\tilde{u}=C \cdot \operatorname{Airy}(\tilde{x})$ for $\tilde{x} \rightarrow \infty$. Typical shapepreserving solutions are shown in Fig. 4. These wave packets are propagating in a self-similar fashion, similar to 


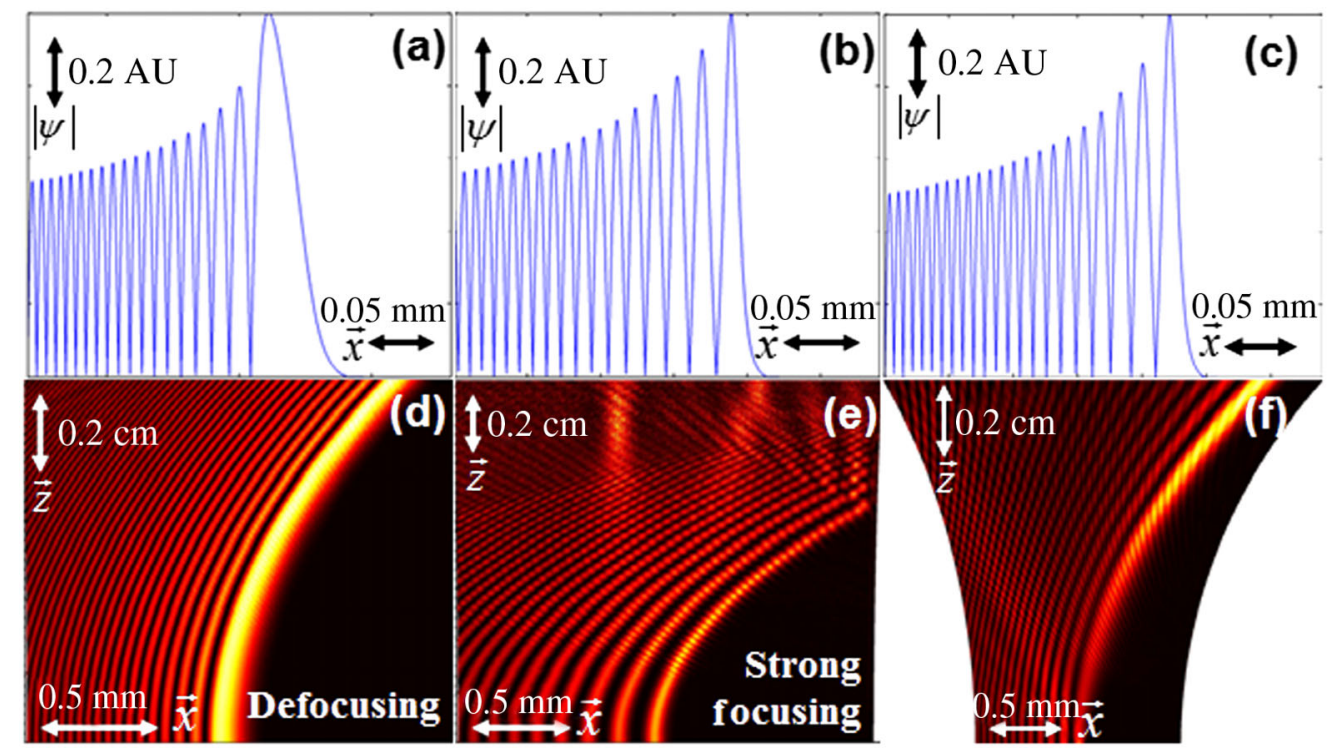

FIG. 4. (a)-(c) Profile of a nonlinear accelerating wave packet in curved space under (a) defocusing and (b), (c) focusing Kerr nonlinearity. The profile differs from the nonlinear accelerating beam: For the defocusing case, the lobes are wider than the linear Airy beam, whereas for the focusing case, the lobes are much thinner. (d)-(f) Evolution of the nonlinear wave packets [of (a)-(c)] on the surface area of a hyperboloid with random noise (of 2\%). (d) Defocusing nonlinearity supports stable propagation, whereas (e) for strong focusing, the beam become unstable to noise. (f) Evolution of the beam of (e) under different surface parameters can stabilize the beam.

their linear counterparts. However, for the focusing case, we find beams with narrower lobes than for the linear beam, and for the defocusing case, we find beams with broader lobes. The solution for the focusing case exists for any $n_{0} C^{2} \kappa / k_{0}^{2}>0$, whereas for the defocusing case, the solution exists only for a finite range of $-2.5<$ $n_{0} \kappa C^{2} / k_{0}^{2}<0$ (in accordance with Ref. [31]). Next, we check the stability of our solutions and find the solution for the defocusing case to be stable under random "white" noise, whereas the self-focusing solutions become unstable after some propagation distance. The most interesting feature is that the stability of the beam depends on the curvature: By changing the parameters of the surface, we can make the beam stable for considerably larger distances (possibly even indefinite ones), as shown in Fig 4. This effect of the curvature on the stability suggests an option for stabilizing nonlinear accelerating beams using the curvature of space, and is directly related to the instability of solitons in negatively curved space [53]. To augment this nonlinear section, we note that other saturable nonlinearities can be handled in a similar fashion, as was done in Ref. [31] for flat space.

The ideas presented here can be implemented in an optical experiment using a thin dielectric layer waveguide on the surface of a 3D shape. The thickness and refractive index of the layer should be chosen to allow only a single mode for $\xi$, which is calculated easily from Eq. (3b). Observing the propagation dynamics of the accelerating beam inside such a $2 \mathrm{D}$ curved-space waveguide surface is possible by examining the input and output beam, by investigating the tails of the modes outside the surface waveguide, and, most importantly, by viewing the light scattered from inhomogeneous material and imperfections inside the surface waveguide or the light fluorescing from it. Moreover, the concepts presented here on accelerating wave packets in curved space are rather general: They can also be used in other optical systems, for example, surface plasmons on curved metallic surfaces. The last decade has witnessed extensive research on the enhancement of the EM fields on metallic shapes, such as spheres and tips, with many applications ranging from commercial products such as near-field microscopes to novel therapeutic applications with small particles $[54,55]$. The ideas we present here allow manipulation of the peak intensity of EM wave packets on curved surfaces by designing the initial conditions (the launch beam). Understanding the dynamics of wave packets in curved space and controlling them by predesigning the launch beam, as presented here, can enable many more new ideas, initiate new therapeutic possibilities, and enable improvement in the enhancement of the EM field for a given geometry. Furthermore, recalling that plasmonic systems always have considerable losses, the wave packets can be designed to compensate for the losses and greatly extend the range for which the main lobe (peak intensity value and shape) remains shape invariant in spite of the losses, as presented in Ref. [56].

Further interesting research will also include the exploration of EM waves in topological surfaces, for example, a doughnut shape. Can we control the trajectory of a wave packet in a surface with a nonzero Chern number just by the design of the initial beam profile? What would be different for a wave packet propagating in a curved space 
with nontrivial topology, for example, crossing the surface of a doughnut from the outside to the center of its hole? These questions are interesting for two reasons: first, because of the extensive interest in topological metrics, such as the known Schwarzschild metric of a black hole, and second, in the spirit of the optical topological effects (photonic topological insulators) observed recently [57].

Before concluding, it is worth discussing the similarities and differences between our findings in curved space and related effects in flat-space metamaterials or photonic potentials in which the EM environment is engineered to correspond to the fictitious force occurring in curved space. In both cases, the behavior of the EM waves can be described by the Maxwell equations in general coordinates, and in both, an optical beam would propagate as if a fictitious force is acting upon it. However, there are some major differences between EM waves in curved space and EM waves in such specifically engineered metamaterials. The principal difference is that in 3D curved-space settings like ours, where the beam is propagating within a thin layer surrounding a 3D volume, the actual "environment" poses restrictions on the EM fields. The simplest example is the one we gave above: Any surface of revolution imposes periodic boundary conditions on the EM field. These conditions underline new solutions of the Maxwell equations: accelerating wave packets that go around the cylindrically symmetric 3D body. Even this simple example does not exist in metamaterials in flat space because there is no way to make the beam go around in flat space under a constant fictitious force as it does in the above examples. Clearly, there are many other examples that are fundamentally even more intriguing. For example, curved space formed by a thin layer surrounding 3D bodies with nontrivial topology, such as the doughnut discussed above, will impose boundary conditions that cannot be implemented in any flat space-even those made of metamaterials. The very fact that the curved space is formed by surrounding an actual 3D body makes a major difference that cannot be imitated in flat space. In the same vein, in our types of geometry, when the 3D body becomes sufficiently narrow, the waves propagating on opposite sides of the body couple to one another, giving rise to new physics that does not exist in flat space.

To summarize, we have found linear and nonlinear, paraxial and nonparaxial, spatially accelerating wave packets in curved space, thereby introducing the concept of accelerating beams to curved-space geometry. The relation of this work to general relativity opens up many ideas for future exploration. For example, one can design a wave front that would be able to compensate for gravitational effects. Indeed, we are currently working on the nonlinear version of this idea, where the accelerating wave packet is what causes the effective curving in space, in an optically nonlinear medium.
This work was supported by the ICORE Excellence Center "Circle of Light," by an Advanced Grant from the European Research Council, by the Israel Ministry of Science and Technology, and by the Israel Science Foundation.

[1] W. G. Unruh, Experimental Black-Hole Evaporation?, Phys. Rev. Lett. 46, 1351 (1981).

[2] R. Schützhold and W. G. Unruh, Gravity Wave Analogues of Black Holes, Phys. Rev. D 66, 044019 (2002).

[3] S. Weinfurtner, E. W. Tedford, M. C. J. Penrice, W. G. Unruh, and G. A. Lawrence, Measurement of Stimulated Hawking Emission in an Analogue System, Phys. Rev. Lett. 106, 021302 (2011).

[4] C. Barceló, S. Liberati, and M. Visser, Probing Semiclassical Analog Gravity in Bose-Einstein Condensates with Widely Tunable Interactions, Phys. Rev. A 68, 053613 (2003).

[5] P. O. Fedichev and U. R. Fischer, Gibbons-Hawking Effect in the Sonic de Sitter Space-Time of an Expanding BoseEinstein-Condensed Gas, Phys. Rev. Lett. 91, 240407 (2003).

[6] O. Lahav, A. Itah, A. Blumkin, C. Gordon, S. Rinott, A. Zayats, and J. Steinhauer, Realization of a Sonic Black Hole Analog in a Bose-Einstein Condensate, Phys. Rev. Lett. 105, 240401 (2010).

[7] R. Schützhold and W. G. Unruh, Hawking Radiation in an Electromagnetic Waveguide?, Phys. Rev. Lett. 95, 031301 (2005).

[8] E. E. Narimanov and A. V. Kildishev, Optical Black Hole: Broadband Omnidirectional Light Absorber, Appl. Phys. Lett. 95, 041106 (2009).

[9] D. A. Genov, S. Zhang, and X. Zhang, Mimicking Celestial Mechanics in Metamaterials, Nat. Phys. 5, 687 (2009).

[10] I. I. Smolyaninov, Surface Plasmon Toy Model of a Rotating Black Hole, New J. Phys. 5, 147 (2003).

[11] I. I. Smolyaninov, Q. Balzano, and C.C. Davis, PlasmonPolaritons on the Surface of a Pseudosphere, Phys. Rev. B 72, 165412 (2005).

[12] U. Leonhardt and P. Piwnicki, Relativistic Effects of Light in Moving Media with Extremely Low Group Velocity, Phys. Rev. Lett. 84, 822 (2000).

[13] T. G. Philbin, C. Kuklewicz, S. Robertson, S. Hill, F. König, and U. Leonhardt, Fiber-Optical Analog of the Event Horizon, Science 319, 1367 (2008).

[14] R. C. T. da Costa, Quantum Mechanics of a Constrained Particle, Phys. Rev. A 23, 1982 (1981).

[15] S. Batz and U. Peschel, Linear and Nonlinear Optics in Curved Space, Phys. Rev. A 78, 043821 (2008).

[16] V. H. Schultheiss, S. Batz, A. Szameit, F. Dreisow, S. Nolte, A. Tünnermann, S. Longhi, and U. Peschel, Optics in Curved Space, Phys. Rev. Lett. 105, 143901 (2010).

[17] M. V. Berry and N. L. Balazs, Nonspreading Wave Packets, Am. J. Phys. 47, 264 (1979).

[18] G. A. Siviloglou and D. N. Christodoulides, Accelerating Finite Energy Airy Beams, Opt. Lett. 32, 979 (2007). 
[19] G. A. Siviloglou, J. Broky, A. Dogariu, and D. N. Christodoulides, Observation of Accelerating Airy Beams, Phys. Rev. Lett. 99, 213901 (2007).

[20] A. Chong, W. H. Renninger, D. N. Christodoulides, and F. W. Wise, Airy-Bessel Wave Packets as Versatile Linear Light Bullets, Nat. Photonics 4, 103 (2010).

[21] D. Abdollahpour, S. Suntsov, D. G. Papazoglou, and S. Tzortzakis, Spatio Temporal Airy Light Bullets in the Linear and Nonlinear Regimes, Phys. Rev. Lett. 105, 253901 (2010).

[22] I. Kaminer, Y. Lumer, M. Segev, and D. N. Christodoulides, Causality Effects on Accelerating Light Pulses, Opt. Express 19, 23132 (2011).

[23] M. A. Bandres, Accelerating Parabolic Beams, Opt. Lett. 33, 1678 (2008).

[24] E. Greenfield, M. Segev, W. Walasik, and O. Raz, Accelerating Light Beams Along Arbitrary Convex Trajectories, Phys. Rev. Lett. 106, 213902 (2011).

[25] I. Chremmos, N. K. Efremidis, and D. N. Christodoulides, Pre-engineered Abruptly Autofocusing Beams, Opt. Lett. 36, 1890 (2011).

[26] L. Froehly, F. Courvoisier, A. Mathis, M. Jacquot, L. Furfaro, R. Giust, P. A. Lacourt, and J. M. Dudley, Arbitrary Accelerating Micron-Scale Caustic Beams in Two and Three Dimensions, Opt. Express 19, 16455 (2011).

[27] R. El-Ganainy, K. G. Makris, M. A. Miri, D. N. Christodoulides, and Z. Chen, Discrete Beam Acceleration in Uniform Waveguide Arrays, Phys. Rev. A 84, 023842 (2011).

[28] Z. Ye, S. Liu, C. Lou, P. Zhang, Y. Hu, D. Song, J. Zhao, and Z. Chen, Acceleration Control of Airy Beams with Optically Induced Refractive-Index Gradient, Opt. Lett. 36, 3230 (2011).

[29] I. Kaminer, J. Nemirovsky, K. G. Makris, and M. Segev, Self-Accelerating Beams in Photonic Crystals, Opt. Express 21, 8886 (2013).

[30] F. Bleckmann, A. Minovich, J. Frohnhaus, D. N. Neshev, and S. Linden, Manipulation of Airy Surface Plasmon Beams, Opt. Lett. 38, 1443 (2013).

[31] I. Kaminer, M. Segev, and D. N. Christodoulides, SelfAccelerating Self-Trapped Optical Beams, Phys. Rev. Lett. 106, 213903 (2011).

[32] R. Bekenstein and M. Segev, Self-Accelerating Optical Beams in Highly Nonlocal Nonlinear Media, Opt. Express 19, 23706 (2011).

[33] A. Lotti, D. Faccio, A. Couairon, D. G. Papazoglou, P. Panagiotopoulos, D. Abdollahpour, and S. Tzortzakis, Stationary Nonlinear Airy Beams, Phys. Rev. A 84, 021807 (2011).

[34] I. Dolev, I. Kaminer, A. Shapira, M. Segev, and A. Arie, Experimental Observation of Self-Accelerating Beams in Quadratic Nonlinear Media, Phys. Rev. Lett. 108, 113903 (2012).

[35] J. Baumgartl, M. Mazilu, and K. Dholakia, Optically Mediated Particle Clearing Using Airy Wavepackets, Nat. Photonics 2, 675 (2008).

[36] P. Zhang, J. Prakash, Z. Zhang, M. S. Mills, N. K. Efremidis, D. N. Christodoulides, and Z. Chen, Trapping and Guiding Microparticles with Morphing Autofocusing Airy Beams, Opt. Lett. 36, 2883 (2011).
[37] P. Polynkin, M. Kolesik, J. V. Moloney, G. A. Siviloglou, and D. N. Christodoulides, Curved Plasma Channel Generation Using Ultraintense Airy Beams, Science 324, 229 (2009).

[38] N. Voloch-Bloch, Y. Lereah, Y. Lilach, A. Gover, and A. Arie, Generation of Electron Airy Beams, Nature (London) 494, 331 (2013).

[39] I. Kaminer, R. Bekenstein, J. Nemirovsky, and M. Segev, Nondiffracting Accelerating Wave Packets of Maxwell's Equations, Phys. Rev. Lett. 108, 163901 (2012).

[40] F. Courvoisier, A. Mathis, L. Froehly, R. Giust, L. Furfaro, P. A. Lacourt, M. Jacquot, and J. M. Dudley, Sending Femtosecond Pulses in Circles: Highly Nonparaxial Accelerating Beams, Opt. Lett. 37, 1736 (2012).

[41] P. Zhang, Y. Hu, D. Cannan, A. Salandrino, T. Li, R. Morandotti, X. Zhang, and Z. Chen, Generation of Linear and Nonlinear Nonparaxial Accelerating Beams, Opt. Lett. 37, 2820 (2012).

[42] I. Kaminer, E. Greenfield, R. Bekenstein, J. Nemirovsky, M. Segev, A. Mathis, L. Froehly, F. Courvoisier, and J. M. Dudley, Accelerating Beyond the Horizon, Opt. Photonics News 23, 26 (2012).

[43] P. Aleahmad, M.-A. Miri, M. S. Mills, I. Kaminer, M. Segev, and D. N. Christodoulides, Fully Vectorial Accelerating Diffraction-Free Helmholtz Beams, Phys. Rev. Lett. 109, 203902 (2012).

[44] P. Zhang, Y. Hu, T. Li, D. Cannan, X. Yin, R. Morandotti, Z. Chen, and X. Zhang, Nonparaxial Mathieu and Weber Accelerating Beams, Phys. Rev. Lett. 109, 193901 (2012).

[45] M. A. Bandres and B. M. Rodríguez-Lara, Nondiffracting Accelerating Waves: Weber Waves and Parabolic Momentum, New J. Phys. 15, 013054 (2013).

[46] L. D. Laundau and E. M. Lifshitz, The Classical Theory Of Fields, (Butterworth-Heinemann, Oxford, England, 1975), 4 th ed.

[47] Utilizing transformation optics techniques to create the effects of accelerating beams as if they propagate on a surface of revolution removes the requirement for periodic boundary conditions, and the solution will be the exact solution of the universal covering space.

[48] We assume propagating solutions. Importantly, the transition to evanescent waves here depends on the metric; namely, any function can become evanescent, at some distance, if the surface is converging [as in Fig. 1(a)], in contrast to flat space where the evanescent nature is predetermined when the spatial frequency equals the wavenumber.

[49] A. Mathis, F. Courvoisier, L. Froehly, L. Furfaro, M. Jacquot, P.-A. Lacourt, and J.-M. Dudley, Micromachining Along a Curve: Femtosecond Laser Micromachining of Curved Profiles in Diamond and Silicon Using Accelerating Beams, Appl. Phys. Lett. 101, 071110 (2012).

[50] M. I. Stockman, Nanofocusing of Optical Energy in Tapered Plasmonic Waveguides, Phys. Rev. Lett. 93, 137404 (2004).

[51] A. Bouhelier, J. Renger, M. R. Beversluis, and L. Novotny, Plasmon-Coupled Tip-Enhanced Near-Field Optical Microscopy, J. Microsc. 210, 220 (2003).

[52] The particular feature of the beam acceleration toward the direction of the other lobes has been observed in plasmonics in flat space by inducing an external linear potential; see F. Bleckmann, A. Minovich, J. Frohnhaus, D. N. Neshev, and 
S. Linden, Manipulation of Airy Surface Plasmon Beams, Opt. Lett. 38, 1443 (2013).

[53] S. Batz and U. Peschel, Solitons in Curved Space of Constant Curvature, Phys. Rev. A 81, 053806 (2010).

[54] B. Hecht, B. Sick, U. P. Wild, V. Deckert, R. Zenobi, O. J. F. Martin, and D. W. Pohl, Scanning Near-Field Optical Microscopy with Aperture Probes: Fundamentals and Applications, J. Chem. Phys. 112, 7761 (2000).

[55] D. Pissuwan, S. M. Valenzuela, and M. B. Cortie, Therapeutic Possibilities of Plasmonically Heated Gold Nanoparticles, Trends Biotechnol. 24, 62 (2006).
[56] R. Schley, I. Kaminer, R. Bekenstein, G. Bartal, and M. Segev," "Loss-Proof Self-Accelerating Plasmons and Exponentially Growing Beams," in Proceedings of CLEO, 2013 (Optical Society of America, 2013), p. QW3N.8, http://www.opticsinfobase.org/abstract.cfm?uri=CLEO_ QELS-2013-QW3N.8.

[57] M. C. Rechtsman, J. M. Zeuner, Y. Plotnik, Y. Lumer, D. Podolsky, F. Dreisow, S. Nolte, M. Segev, and A. Szameit, Photonic Floquet Topological Insulators, Nature (London) 496, 196 (2013). 